\title{
Uso de la Eisenia hortensis (lombriz de tierra) en el vermicompostaje de residuos orgánicos
}

\section{Use of Eisenia hortensis (earthworm) in the vermicomposting of organic waste}

\author{
Annick E Huaccha $\mathrm{C}^{1}$, Franklin H Fernández Z $\mathrm{Z}^{2}$, Sergio H Quiroga $\mathrm{R}^{3}$ y Bárbara Álvarez B ${ }^{4}$.
}

\begin{abstract}
RESUMEN
Actualmente, el paradigma de la economía circular es cada vez más importante, de ahí la necesidad de abordar los crecientes desafíos relacionados con la prosperidad económica, la protección del ambiente y la prevención de la contaminación, facilitando así el desarrollo sostenible. La gestión de residuos se ha convertido en un imprescindible de cara a alcanzar la mayor sostenibilidad posible. La gestión de los residuos orgánicos mediante el vermicompostaje es una biotecnología con grandes beneficios ambientales y de bajo costo. En este proyecto se ha evaluado la capacidad que tiene la lombriz Eisenia hortensis denominada también Dendrobaena veneta para digerir restos de frutas y verduras, y estiércol de caballo pre-compostado. En todas las evaluaciones $E$. hortensis demostró tener la capacidad de generar modificaciones fisicoquímicas sobre los residuos iniciales a través sobre todo de procesos de mineralización.
\end{abstract}

Palabras clave: Eisenia hortensis, vermicompostaje, residuos orgánicos.

\begin{abstract}
Currently, the paradigm of the circular economy is increasingly important, hence the need to address the growing challenges related to economic prosperity, environmental protection and pollution prevention, thus facilitating sustainable development. Waste management has become essential in order to achieve the greatest possible sustainability. The management of organic waste through vermicomposting is a biotechnology with great environmental benefits and low cost. This project has evaluated the capacity of the worm Eisenia hortensis also called Dendrobaena veneta to digest fruit and vegetable remains, and horse manure pre-composted. In all the evaluations, $E$. hortensis demonstrated the ability to generate physico-chemical modifications on the initial residues through, above all, mineralization processes.
\end{abstract}

Keywords: Eisenia hortensis, vermicomposting, organic waste.

\footnotetext{
${ }^{1}$ Ingeniera ambiental. Universidad Nacional de Jaén, Cajamarca, Perú. Email: annick huaccha@ unj.edu.pe

${ }^{2}$ Ingeniero forestal y ambiental. Universidad Nacional de Jaén, Cajamarca, Perú. Email: fran.9615fernandez@gmail.com

${ }^{3}$ Ingeniero agrónomo. Empresa Ecocelta Galicia, España. Email: squiroga@ecocelta.com

${ }^{4}$ Licenciada en biología. Empresa Ecocelta Galicia, España. Email: 1aboratorio@ecocelta.com
} 


\section{INTRODUCCIÓN}

El Ministerio del Ambiente (MINAM) de Perú, viene desarrollando soluciones innovadoras con el fin de mejorar la calidad ambiental del país incorporando la gestión de los residuos sólidos; para esto ha elaborado el Plan Nacional de Gestión Integral de Residuos Sólidos 2016-2024 (PLANRES), en el que se plasma la situación actual de los residuos sólidos en el país.

Según los datos reportados por el MINAM (2014), el Perú, en el año 2014, generó 7497482 t/año de residuos urbanos municipales, de éstos; el $64 \%$ son residuos domiciliarios y el $26 \%$ son residuos no domiciliarios; en el reporte señala que el $52 \%$ de los residuos sólidos son materia orgánica, el $19 \%$ son residuos no aprovechables y el $29 \%$ engloba a los residuos aprovechables y reciclables. Estos datos, revelan la gran cantidad de materia orgánica generada en el país, que bien puede ser tratada mediante sistemas de compostaje y vermicompostaje.

Moreno et al. (2014) definen al vermicompostaje como un proceso de biooxidación, degradación y estabilización de la materia orgánica mediada por la acción combinada de lombrices y microorganismos bajo condiciones aerobias y mesófilas, con el que se obtiene un producto final estabilizado; mientras que Edwars (1988) señala que, el verrmicompostaje es un proceso biotecnológico que permite degradar y estabilizar residuos orgánicos bajo condiciones aerobias y mesófilas mediante la acción de ciertas especies de lombrices de tierra capaces de alimentarse del residuo a la vez que aceleran su degradación microbiana.

Según Domínguez y Gómez (2010) indican que, Eisenia hortensis o Dendrobaena veneta es una lombriz de tipo epígeo, cuyas características metabólicas que favorecen su adaptación a todo tipo de cambios. Estos organismos son hermafroditas y alcanzan la madurez sexual a los 65 días.

Garg et al. (2008) afirman que las especies de lombrices involucradas en el proceso de vermicompostaje son capaces de ingerir al día cantidades de residuos equivalentes al 50-100 \% de su propio peso; la descomposición de los residuos orgánicos por lombrices se ve favorecida por la acción de microorganismos endosimbiontes de su intestino, estos producen enzimas extracelulares que degradan celulosa y compuestos fenólicos, aumentando la mineralización del carbono y nitrógeno del material ingerido (Domínguez et al., 2009).

Domínguez (2004) señala que, el movimiento de las lombrices a través del residuo del que se alimentan promueve su aireación estimulando su bioxidación microbiana, de esta forma, debido al trabajo conjunto entre lombrices y microorganismos acción conjunta de lombrices y microorganismos el residuo orgánico es degradado hasta mineralizarse parcialmente, humificarse y estabilizarse.

Según Singh et al. (2011) señalan que, la tecnología de vermicompostaje en la gestión de los residuos orgánicos convencionales y no convencionales, ha crecido considerablemente como resultado de grandes avances científicos en varias partes del mundo, esta tecnología se basa en el 
hábito de alimentación detritívoro de algunas especies de lombrices, organismos capaces de colonizar una gran variedad de sustratos orgánicos tal como lo indica Mamani et al. (2012).

Cabanilla et al. (2013) indican que, la generación de residuos sólidos orgánicos ha impulsado la búsqueda de alternativas para su tratamiento y disposición final, siendo una de ellas, la producción de humus de lombriz como propuesta ecológica al uso desmedido de fertilizantes químicos.

Bajo este contexto, se ha evaluado la capacidad que tiene la lombriz Eisenia hortensis denominada también Dendrobaena veneta para digerir restos de frutas, verduras, y estiércol de caballo precompostado.

\section{MATERIALES Y MÉTODOS}

\section{Área de estudio}

La investigación está situada geográficamente en 540204.4 E, 4673709.31 N, fue desarrollada en las instalaciones de la empresa Ecocelta Galicia S. L, ubicada en el municipio de Pontareas, provincia de Pontevedra, comunidad autónoma de Galicia, España, toda la investigación fue desarrollada bajo condiciones controladas, siendo desarrollada en invierno, entre enero y marzo de 2019.

\section{Materiales}

1. Sustratos

- Estiércol de caballo (incluye paja).

- Restos de frutas y verduras.

2. Material biológico: Individuos de E. hortensis adultas (con clitelo bien desarrollado), los que fueron colectados de una población identificada y adaptada a la vermigestión de residuos orgánicos.

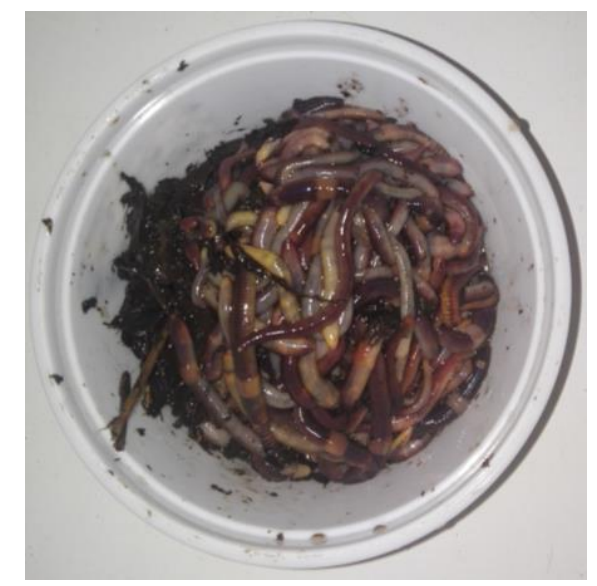

Figura 1: Individuos de E. hortensis adultas, listos para ser inoculados en sustrato de prueba. 


\section{Métodos}

- Selección de sustratos para el vermicompostaje: esta selección de sustratos se realizó mediante el estudio bibliográfico de los residuos con más necesidad de ser gestionados mediante métodos biológicos y sostenibles.

- Alimentación gradual: se adicionó capas delgadas del nuevo sustrato sobre un lecho que contenía población establecida de lombrices.

- Adaptación del sustrato: se realizó a través del proceso de pre-compostaje aplicado al material que fue empleado para alimentar a las lombrices, pasando por un periodo previo de estabilización, para posteriormente ser mezclado con otros subproductos para el cambio de las propiedades fisicoquímicas del material.

- Adición de $255 \mathrm{~g}$ de lombrices de E. hortensis a cada sustrato. Los ensayos se mantuvieron por 60 días.

- Evaluación del proceso, pH, temperatura, conductividad, biomasa, eficiencia de transformación del abono, densidad de los sustratos, influencia de los sustratos sobre los parámetros químicos del vermicompost.

\section{RESULTADOS Y DISCUSIÓN}

\section{Biomasa}

Bajo este parámetro se analizó la cantidad de biomasa expresada como gramos de lombriz existentes por gramo de sustrato.

Tabla 1. Biomasa (g de lombriz/g de sustrato) a lo largo del vermicompostaje de estiércol de caballo, frutas y verduras con la especie de lombriz de tierra E. hortensis.

\begin{tabular}{lcc}
\hline Tratamiento & Día 0 & Día 60 \\
\hline Estiércol de caballo & 12.22 & 14.08 \\
Frutas y verduras & 18.64 & 30.32 \\
\hline
\end{tabular}

Como se puede comprobar en la Tabla 1, los mayores valores de biomasa tuvieron lugar para las poblaciones alimentadas con restos de frutas y verduras, debido a que, la reducción en el peso del sustrato al final del ensayo es mucho mayor que el que se dio en el de estiércol de caballo; del mismo modo lo señala Álvarez (2017) en su estudio donde determinó que los mayores valores de biomasa tuvieron lugar para las poblaciones alimentadas con restos de comida lo cual se podría 
deber a que la reducción en el volumen del sustrato al final del ensayo es mucho mayor que el que se dio en los demás tipos de restos (estiércol de caballo y purín de cerdo).

\section{Eficiencia de transformación del abono (EFTA)}

Tabla 2. Balance de masas a lo largo del vermicompostaje de estiércol de caballo, frutas y verduras, comida con la especie de lombriz de tierra E. hortensis.

\begin{tabular}{lccc}
\hline Tratamiento & $\begin{array}{c}\text { Sustrato } \\
\text { suministrado }\end{array}$ & $\begin{array}{c}\text { Sustrato } \\
\text { cosechado }\end{array}$ & EFTA \\
\hline Estiércol de caballo & 20.86 & 18.10 & 86.77 \\
Frutas y verduras & 13.68 & 8.41 & 61.50 \\
\hline
\end{tabular}

La eficiencia de transformación del abono es un parámetro que se emplea para representar el grado de reducción, o de transformación del alimento suministrado inicialmente a las pruebas de vermicompostaje. De acuerdo con la Tabla 2, la EFTA fue mucho mayor en los tratamientos con caballo; este resultado tal como los señala Edwars (1988), se debe a las características físicas y químicas de los sustratos usados, además, Paredes et al. (2000) y Edwars (1988) afirman que, al mineralizarse la materia orgánica se volatiliza y ello se traduce en una mayor reducción del peso y volumen del sustrato, que deriva en un mayor valor de EFTA.

\section{Densidad de los sustratos}

Tabla 3. Densidad g/L de las pruebas a lo largo del vermicompostaje de estiércol de caballo, frutas y verduras con la especie de lombriz de tierra E. hortensis.

\begin{tabular}{lcc}
\hline \multicolumn{1}{c}{ Tratamiento } & Día 0 & Día 60 \\
\hline Estiércol de caballo & 0.60 & 0.63 \\
Frutas y verduras & 0.39 & 1.04 \\
\hline
\end{tabular}

La relación que existe entre el peso del material (g) y el volumen que ocupa (L) se conoce como densidad y se trata de un concepto de gran interés y utilidad práctica al momento de poner en marcha un proceso productivo de vermicompostaje. La medición de este valor a lo largo de los 60 días permite evaluar de forma indirecta ciertas características del sustrato, siendo las más importantes, la porosidad y la humedad. En los procesos de compostaje y vermicompostaje lo habitual es que los sustratos incrementen su densidad a medida que son procesados, debido sobre todo a la disminución del tamaño de la partícula y a una mayor concentración de nutrientes. 


\section{Influencia de los sustratos sobre los parámetros químicos del vermicompost}

Tabla 4: Influencia de los sustratos sobre los parámetros químicos del vermicompost

\begin{tabular}{|c|c|c|c|}
\hline \multicolumn{2}{|c|}{ Tratamiento } & \multirow{2}{*}{$\begin{array}{r}\text { Día } 0 \\
8.13\end{array}$} & \multirow{2}{*}{$\begin{array}{r}\text { Día } 60 \\
7.50\end{array}$} \\
\hline Ectiércolde cahal & $\mathrm{pH}$ & & \\
\hline Estercor de cadallo & Conductividad (uS) & 1284.00 & 1153.00 \\
\hline \multirow{2}{*}{ Frutas y verduras } & $\mathrm{pH}$ & 4.40 & 7.19 \\
\hline & Conductividad (uS) & 1063.00 & 2100.00 \\
\hline
\end{tabular}

Los parámetros químicos son determinantes a la hora de dar salida al producto final obtenido en procesos de vermicompostaje. Los valores obtenidos a los 60 días para los vermicompost obtenidos en los dos tratamientos son de gran relevancia para la constitución de unas conclusiones válidas y consolidadas.

El pH es uno de los parámetros más importante para definir la capacidad fertilizante de un vermicompost. La alcalinidad o acidez de un suelo influye en la disponibilidad de los nutrientes de forma muy relevante. El valor ideal para el $\mathrm{pH}$ en suelos es en torno a 6 - 7 dependiendo de las especies que se quieran cultivar.

En los restos de frutas y verduras se pasa de un pH ácido (4.40) a casi neutro (7.19), encontrándose este dentro del rango óptimo para la mayor parte de los cultivos $(6.00-7.00)$. En el estiércol de caballo se pasa de un pH neutro (8.13) a básico (7.50), convirtiéndolo en un producto fertilizante con un pH ideal para la neutralización de los suelos. Detrás de estos aumentos de pH se encuentra el proceso de mineralización de la materia orgánica. Durante el vermicompostaje, tiene lugar la alcalinización del medio debido a la generación de amonio y degradación de ácidos orgánicos por la actividad de organismos aerobios. También es importante la producción de ácidos húmicos a partir de la materia orgánica, que asegura la neutralización del medio, compensando a los compuestos básicos generados en esta fase (Richard, 1996; Sánchez et al., 2001).

\section{CONCLUSIONES}

La biomasa expresada en $\mathrm{g}$ de lombriz/ $\mathrm{g}$ de sustrato, es mayor para el sustrato compuesto por frutas y verduras, esto debido a la disminución de peso en el sustrato final.

Los datos de las pruebas fisicoquímicas nos muestran cambios en los sustratos tras ser vermicompostados durante 60 días, haciendo notar los procesos de mineralización de la materia orgánica y los nutrientes. 
Los resultados obtenidos en la eficiencia de transformación del abono expresan que, para el estiércol de caballo tiene una EFTA mayor, comparada con la EFTA del sustrato de frutas y verduras, además se evidencia un incremento de la densidad de los sustratos, siendo mayor en el sustrato de frutas y verduras.

\section{REFERENCIAS BIBLIOGRÁFICAS}

Bernal, M., Alburquerque, J. y Moral, R. (2009). Composting of animal manures and chemical criteria for compost maturity assessment. A review.Bioresourcetechnol. 100: 5444-5453.

Bollo, E. (2001). Lombricultura, una alternativa de reciclaje. Soboc Grafic, Quito, Ecuador. 158 p.

Cabanillas, C.; Tablada, M. and Ledesma, A. (2013). Vermicompost: alternative to urea in basil seed production. Management of Environmental Quality International Journal. 24:165- 177.

Cabildo, M., Claramunt, R., Comago, M., Escolástico, C., Esteban, S., Farrán, M., García, M., López, C., Pérez, J., Pérez, M., Santamaría, M., Sanz, D. (2008). Tratamiento de la materia orgánica. Compostaje, pp. 141-165. In: Reciclado y tratamiento de residuos. UNED. Ciencias Ambientales. Madrid.

Chaudhari, R., Datar, M. y Babookani, M. (2011) Municipal solid waste management through vermicomposting employing exotic species of earthworm Eudriluseugeniae. J EnvironSciEng.53: 29-34.

Dmitriew, C. y Rowe, L. (2007) Effects of early resource limitation and compensatory growth on lifetime fitness in the ladybird beetle (Harmonia axyridis). J EvolutionBio. 120: 1298-1310.

Dominguez, J. y Edwards, C. (2011) Chapter 2 Relationships between Composting and Vermicomposting. Vermiculture Technology: Earthworms, Organic Wastes, and Environmental management CRC Press, Florida USA. 11-25 (B).

Dominguez, J. y Gómez-Brando, M. (2010). Ciclos de vida de las lombrices de tierra aptas para el vermicompostaje. Acta Zool. Mex. 2: 309-320.

Domínguez, J.; Aira, M. y Gómez, B. (2009). El papel de las lombrices de tierra en la descomposición de la materia orgánica y el ciclo de nutrientes. Ecosistemas. 18(2):20-31.

Dominguez, J. (2004). State-of-the-Art and New Perspectives on Vermicomposting Research. Edwards C. EarthwormEcology. CRC PressFlorida USA. 401-4024 (A).

Duran, L. y Henriquez, C. (2009) Crecimiento y Reproduccón de la Lombriz Roja (Eiseniafoetida) en cinco sustratos orgánicos. AgronCostarric33: 275-281 Edwards. 
Edwards, C. (1988). Breakdown of animal, vegetable and industrial organic wastes by earthworms., ed. C. A. Edwards and E. F. Neuhauser, In Earthworms in Waste and Environmental Management, The Hague, the Netherlands: 21-31.

Fayolle, L, Michaud, H. y Cluzeau, D. (1997) Stawiecki, JInfluence of temperatura and food source on the life cycle of the earthworm Soil BiolBiochem. 29: 747-750.

Garg, V., Gupta, R., Yadav, A. (2008). Potential of vermicomposting technology in solid waste management, in: Pandey, A., Soccol, C.R., Larroche, C. (Eds.), Current Developments in Solid-state Fermentation. Springer New York468-511.

Garg, V., Chand, S., Chhillar, A. y Yadavgrowth, A. (2005) Grow and reproduction of eiseniafoetidaIn various animal wastes during vermicomposting. ApplEcolEnv Res. 3: 51-59.

Godínez, E., Lagunes, J., Corona, J. y Barajas, M. (2017). Growth and reproductive potential of Eiseniafoetida (Sav) on various zoo animal dungs after two methods of pre-composting followed by vermicomposting WasteManage.

Gunadi, B. y Edwards, C. (2003): The effect of multiple applications of different organic wastes on the growth, fecundity and survival of Eiseniafoetida (Savigny) (Lumbricidae). Pedobiologia. 47: 321-330.

James, S. y Hendrix, P. (2004). Invasion of exotic earthworms into North America and other regions. In: Edwards, C.A.(Ed.), Earthworm Ecology, 2nd ed., CRC Press LLC, Boca Raton, FL, pp. 75-88.

Lara, A. y Quintero, R. (2006). Manual de producción de humus de lombriz. Unidad Académica de Agronomía. Universidad Autónoma de Zacatecas "Francisco García Salinas”. Campus Montecillo, Colegio de Postgraduados en Ciencias Agrícolas. Montecillo, Edo. de México, México. Fundación Produce Zacatecas, A. C. 43 p.

Lazcano, C., Gómez, M. y Domínguez, J. (2008). Comparison of the effectiveness of composting and vermicomposting for the biological stabilization of cattle manure. Chemosphere 72 : 1013-1019 Loehr.

Mamani, M.; Mamani, P.; Sainz, M. y Villca, H. R. (2012). Comportamiento de la lombriz roja (Eisenia spp.) en sistemas de vermicompostaje de residuos orgánicos. J. Selva Andina Res. Soc. 1(1):44-54.

Ministerio del Ambiente (2014). Sexto Informe Nacional de Residuos Sólidos Municipales y no Municipales 2013 Lima, Perú. Disponible http://redrrss.minam.gob.pe/material/20160328155703.pdf 
Monedero, M., Roig, A., Paredes, C. y Bernal, M. (2001). Nitrogen transformation during organic waste composting by the Rutgers system and its effects on $\mathrm{pH}, \mathrm{EC}$ and maturity of the composting mixtures. Biores. Technol., 78 301-308.

Moreno, A. (2005). Origen, importancia y aplicación de la vermicomposta para el desarrollo de especies vegetales. Revista Agraria Nueva Época 2(3):15-23.

Nagavallemma, K., Wani, S., Lacroix, S., Padmaja, V., Vineela, C., Rao, M. y Sahrawat, K., (2004) Vermicomposting: Recycling Wastes into Valuable Organic Fertilizer.Global Theme on Agroecosystems Report $n^{\circ}$. 8. Monograph. International Crops Research Institute for the Semi-Arid Tropics, Patancheru, Andhra Pradesh, India.

OECD (2015), Environment at a Glance 2015: OECD Indicators, OECD Publishing, Paris Onwosi, C., Igbokwe, V., Odimba, J., Eke, I., Nwankwoala, M. Iroh, I., Ezeogu, L. (2017) Composting technology in waste stabilization: On the methods, challenges and future prospects. J EnvironManag. 190: 140-157.

Paredes, C., Roig, A., Bernal, M., Sánchez, M. y Cegarra, J., (2000). Evolution of organic matter and nitrogen during co-composting of olive mil wastewater with solid organic wastes. Biol. Fertil. Soils 32, 222-227.

Reinecke, A. y Viljoen, S. (1990). The influence of feeding patterns on growth and reproduction of the vermicomposting earthworm Eiseniafetida (Oligochaeta). Biol. Fertil. Soils. 10: 184-187.

Richard, T. (1996). The Effect of Particle Size on Bioavailability. NY: CornellCompostingScience and Engineering.

Sánchez, M., Roig, A., Paredes, C. y Bernal, M. (2001). Nitrogen transformation during organic waste composting by the Rutgers system and its effects on $\mathrm{pH}, \mathrm{EC}$ and maturity of the composting mixtures. Biores. Technol., 78 301-308.

Schuldt, M. (2004). Lombricultura fácil. Work Graf, La Plata 153 p.

Singh, P.; Singh, P.; Araujo, S.; Ibrahim, H. and Sulaiman, O. (2011). Management of urban solid waste: Vermicomposting a sustainable option. Res. Conserv. Recy. 55(7):719-729.

Vázquez, M. y Soto, M. (2017). The efficiency of home composting programmes and compost quality. Waste Manage. 